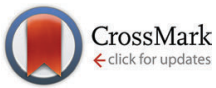

Cite this: Phys. Chem. Chem. Phys. 2016, 18, 28140

Received 26th July 2016, Accepted 20th September 2016 DOI: $10.1039 / c 6 c p 05205 c$

www.rsc.org/pccp

\title{
Bottom-up excited state dynamics of two cinnamate-based sunscreen filter molecules $\uparrow$
}

\author{
Yoann Peperstraete, ${ }^{a b}$ Michael Staniforth, ${ }^{a}$ Lewis A. Baker, ${ }^{a}$ \\ Natércia D. N. Rodrigues, ${ }^{a}$ Neil C. Cole-Filipiak, ${ }^{a}$ Wen-Dong Quan ${ }^{a}$ and \\ Vasilios G. Stavros*a
}

\begin{abstract}
Methyl-E-4-methoxycinnamate (E-MMC) is a model chromophore of the commonly used commercial sunscreen agent, 2-ethylhexyl-E-4-methoxycinnamate (E-EHMC). In an effort to garner a molecular-level understanding of the photoprotection mechanisms in operation with $E$-EHMC, we have used time-resolved pump-probe spectroscopy to explore E-MMC's and E-EHMC's excited state dynamics upon UV-B photoexcitation to the $S_{1}\left(1^{1} \pi \pi^{\star}\right)$ state in both the gas- and solution-phase. In the gas-phase, our studies suggest that the excited state dynamics are driven by non-radiative decay from the $1^{1} \pi \pi^{\star}$ to the $S_{3}\left(1^{1} n \pi^{\star}\right)$ state, followed by de-excitation from the $1^{1} n \pi^{\star}$ to the ground electronic state $\left(S_{0}\right)$. Using both a non-polaraprotic solvent, cyclohexane, and a polar-protic solvent, methanol, we investigated E-MMC and E-EHMC's photochemistry in a more realistic, 'closer-to-shelf' environment. A stark change to the excited state dynamics in the gas-phase is observed in the solution-phase suggesting that the dynamics are now driven by efficient $E / Z$ isomerisation from the initially photoexcited $1^{1} \pi \pi^{\star}$ state to $S_{0}$.
\end{abstract}

\section{Introduction}

Ultraviolet (UV) light has been shown to cause photodamage to living organisms and is responsible for a range of adverse effects, including mutations to DNA. ${ }^{1-4}$ As such, natural selection has resulted in many different photoprotection mechanisms against this harmful radiation, such as negative phototaxis for bacteria ${ }^{5-7}$ or the presence of UV absorbing sunscreen molecules in plants ${ }^{8,9}$ to name but a few. The key absorbing sites of these molecules, or chromophores, have been shown to play a central role in photoprotection. ${ }^{10-14}$ Consequently, the photochemistry and photophysics of these, and related chromophores, have received considerable attention, both theoretically ${ }^{14,15}$ and experimentally, in both the gas- ${ }^{11,12,16-19}$ and solution-phase. ${ }^{10,13,20}$

Many of these chromophores are cinnamate-based derivatives which, as well as serving in natural photoprotection, have also found extensive use in artificial photoprotection. For example, 2-ethylhexyl-E-4-methoxycinnamate ( $E$-EHMC, see structure in Fig. 1b) is an organic sunscreen filter commonly found in

\footnotetext{
${ }^{a}$ University of Warwick, Gibbet Hill Road, Coventry, CV4 7AL, UK. E-mail:v.stavros@warwick.ac.uk

${ }^{b}$ ENS de Cachan, 61 Avenue du Président Wilson, 94230, Cachan, France

$\dagger$ Electronic supplementary information (ESI) available: Molecular structures of MMC, instrument response determination, kinetic analysis of gas- and solutionphase spectra, attempted biexponential TR-IY transient fits, TR-IY transient with $315 \mathrm{~nm}$ probe, detailed difference spectrum in methanol, further details of global fitting, NMR measurements, and TAS line-outs at $450 \mathrm{~nm}$. See DOI: 10.1039/ c6cp05205c
}

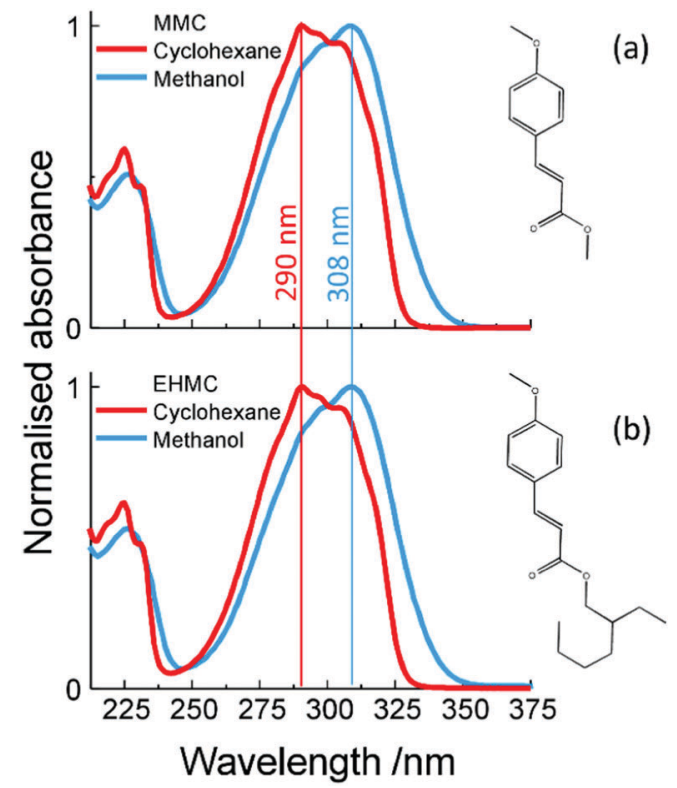

Fig. 1 UV-visible steady static absorption spectra in cyclohexane (red lines) and methanol (blue lines) of (a) E-MMC, and (b) E-EHMC, see insets for molecular structures. The absorption maxima are indicated by the coloured vertical lines.

commercial sunscreens. Surprisingly however, while numerous studies have been performed on the photoprotection mechanisms in operation in E-EHMC, very little is known about the dynamical processes that ensue shortly after photoexcitation 
(femtosecond, fs, to picosecond, ps, timescale). In the present study, we use a 'bottom-up' approach ${ }^{21}$ as a vehicle for understanding the ultrafast processes in operation in $E$-EHMC following UV irradiation, which may provide crucial insight into its widespread use as a sunscreen filter molecule. As a stepping stone towards our goal and in accord with our bottom-up thesis, we also study methyl-E-4-methoxycinnamate (E-MMC, Fig. 1a). This is a building block of $E$-EHMC which, as with $E$-EHMC, displays a broadband absorption centred in the UV-B region (280-315 nm) as depicted in Fig. 1.

Numerous spectroscopic studies have been carried out on $E$-EHMC and $E$-MMC. Through frequency resolved studies, Tan et $a .^{22}$ observed the presence of two rotamers of $E$-MMC and $E$-EHMC in the gas-phase (see structures in the ESI $\dagger$ ). From their spectral line width measurements, they inferred sub-ps and ps $(\sim 2 \mathrm{ps})$ lifetimes for the initially excited $S_{1}\left(1^{1} \pi \pi^{*}\right)$ state of $E$-EHMC and $E$-MMC, respectively. These time-constants were assigned to non-radiative decay to an adiabatically lower $S_{3}\left(1^{1} n \pi^{*}\right)$ state. As observed by the same authors through pump-probe spectroscopy, in the nanosecond (ns) regime, this $1^{1} \mathrm{n} \pi^{*}$ state is a long-lived state in both $E$-MMC (24 ns) and $E$-EHMC (17.7 ns). Interestingly, upon microsolvation by addition of one water molecule to $E$-MMC, the ns lifetime vanished. This was attributed to the $1^{1} \mathrm{n} \pi^{*}$ state increasing in energy upon microsolvation, ${ }^{23}$ leading to an alternative and rapid non-radiative decay mechanism from the $1^{1} \pi \pi^{*}$ state to the ground electronic state, $\mathrm{S}_{0}$, likely mediated by $\mathrm{C}=\mathrm{C}(E / Z)$ bond isomerisation. Since then, $E / Z$ isomerisation of MMC has been observed in rare gas matrices. ${ }^{24}$ Moreover, the isomerisation mechanism has been verified by more recent $a b$ initio calculations performed on MMC and $\mathrm{MMC}-\mathrm{H}_{2} \mathrm{O}$ clusters. ${ }^{25}$ These calculations also suggest that two mechanisms are in competition: $E / Z$ isomerisation through a $1^{1} \pi \pi^{*} / S_{0}$ conical intersection (CI) and internal conversion (IC) through an analogous $1^{1} \pi \pi^{*} / 1^{1} \mathrm{n} \pi^{*} \mathrm{CI}$. In $E$-MMC, contrary to $E$-MMC- $\mathrm{H}_{2} \mathrm{O}$, the $1^{1} \pi \pi^{*} \rightarrow \mathrm{S}_{0}$ transition barrier is energetically higher than that for $1^{1} \pi \pi^{*} \rightarrow 1^{1} n \pi^{*}$. This change in barrier height is caused by the relative change in energies of the $1^{1} \pi \pi^{*}$ and $1^{1} \mathrm{n} \pi^{*}$ states, effectively switching the order of their local minima. Indeed, the change in shape between the absorption spectra shown in Fig. 1 suggests such a shift in ordering of the lowest lying excited states when going from a non-polar to a polar, hydrogen bonding solvent. These findings accord with the experiments by Tan et al.; IC via $1^{1} \pi \pi^{*} \rightarrow 1^{1} \mathrm{n} \pi^{*}$ dominates in $E$-MMC whilst for $E$-MMC- $\mathrm{H}_{2} \mathrm{O}$, IC via $1^{1} \pi \pi^{*} \rightarrow \mathrm{S}_{0}$ dominates.

Due to the ns temporal resolution of the experiments by Tan et al., these studies were unable to provide a complete description of the ultrafast dynamics of photoexcited E-MMC. Miyazaki et al. ${ }^{26}$ carried out a time-resolved pump-probe study with a 12 ps timeresolution. They observed an energy-dependence in the lifetime of the photoexcited E-MMC: timescales decreased from 280 to 13 ps with increasing excitation energy into the $1^{1} \pi \pi^{*}$ state, which ranged from 32328 to $33155 \mathrm{~cm}^{-1}$. However, the $12 \mathrm{ps}$ timeresolution of their experiment meant that they were unable to resolve the 2 ps component of $E$-MMC, as inferred by Tan et $a l^{22}$

Pump-probe spectroscopy experiments of sunscreen filter molecules, in both gas- ${ }^{17,22,27}$ and solution-phase, ${ }^{13,20,28,29}$ have proven crucial towards our understanding of how these vitally important molecules may protect us against deleterious UV radiation exposure. Our goal in the present work was to directly probe the $1^{1} \pi \pi^{*} \rightarrow 1^{1} \mathrm{n} \pi^{*}$ IC in $E$-EHMC to offer further insight into the 2 ps and sub-ps components inferred from prior measurements. To do so, we probed the relaxation mechanism of UV-B photoexcited $E$-MMC and $E$-EHMC using fs time-resolved ion yield (TR-IY) spectroscopy in the gas-phase. Moreover, as it has been shown, gas-phase results can prove highly informative to the interpretation of the solution-phase counterparts. ${ }^{30,31}$ Thus, with reference to previously reported theoretical calculations ${ }^{25}$ on MMC and MMC- $\mathrm{H}_{2} \mathrm{O}$, we extended the microsolvation studies by Tan et al., ${ }^{22}$ to the solution-phase, in both a non-polar-aprotic and polar-protic solvent, utilising transient electronic (UV-visible) absorption spectroscopy (TEAS).

\section{Experimental methods}

\subsection{Gas-phase setup}

The experimental setup has been described in detail elsewhere. ${ }^{32}$ Briefly, and with detail pertaining to the present measurements, E-MMC (Fluorochem, >95\%) or E-EHMC (Sigma-Aldrich, 98\%, stabilised with 500 to 1000 ppm of butylated hydroxytoluene), heated to $130-150{ }^{\circ} \mathrm{C}$, is seeded in 3 bar of He. It is then expanded into vacuum ( $\sim 10^{-7}$ mbar) to create a molecular beam via an Even-Lavie pulsed solenoid valve, ${ }^{33}$ operating at $125 \mathrm{~Hz}$. Two UV laser pulses ( $\sim 100$ fs duration and $\sim 500 \mathrm{~cm}^{-1}$ bandwidth), a pump and a probe, intersect the molecular beam. To obtain these two laser pulses, the fundamental $800 \mathrm{~nm}$ laser beam (40 fs, $3 \mathrm{~mJ}$ per pulse), outputted from a commercial Ti:Sapphire regenerative amplifier (Spectra-Physics, Spitfire XP), is split into three equal components ( $1 \mathrm{~mJ}$ per pulse), two of which are used in the present gas-phase experiments. The first pulse is used to pump an optical parametric amplifier (TOPAS) which provides the tunable UV pump pulse used to promote the molecule of interest to the $1^{1} \pi \pi^{*}$ excited state: $\lambda_{\text {pump }}=290 \mathrm{~nm}$ or $\lambda_{\text {pump }}=$ $308 \mathrm{~nm}(\sim 0.5 \mu \mathrm{J}$ per pulse for both). These wavelengths have been chosen to be consistent with the peak UV-B absorption in the steady state absorption spectra for $E$-MMC and E-EHMC in cyclohexane and methanol, shown in Fig. 1. The second pulse is used to generate the probe laser beam of $200 \mathrm{~nm}$ by sending the $800 \mathrm{~nm}$ beam through three successive (Type I, Type II and Type I) $\beta$-barium borate crystals. The polarisation of the probe is held at magic angle $\left(54.7^{\circ}\right)$ relative to the pump polarisation, using a half wave plate. The time delay between the pump and the probe $(\Delta t)$ is controlled using a gold retroreflector mounted on a delay stage situated along the probe beam-path. The wavelength of the probe is chosen to ensure one-photon ionisation of the photoexcited molecules. The ions created in the molecular beam are then accelerated and detected in a time-of-flight mass spectrometer, which allows us to obtain pump-probe transients of a given mass-to-charge species; here we focus solely on the parent cations, $E$-MMC ${ }^{+}$and $E$-EHMC ${ }^{+}$. The current pump-probe setup provides a temporal resolution of $\sim 150 \mathrm{fs}$ (see ESI $\dagger$ for details). 


\subsection{Solution-phase setup}

The solution-phase experimental setup has also been described previously $^{34,35}$ and is briefly summarised here. For all TEAS measurements, $\sim 0.1 \mathrm{mM}$ solutions of E-MMC (Fluorochem, $>95 \%$ ) or E-EHMC (Sigma-Aldrich, 98\%), in either cyclohexane (VWR, $>99 \%$ ) or methanol (Sigma-Aldrich, 99.6\%), were recirculated through a flow cell (Harrick Scientific), made of two $\mathrm{CaF}_{2}$ windows and $100 \mu \mathrm{m}$ thick PTFE spacers. Background subtraction is performed by recording the absorbance spectra of irradiated and non-irradiated sample by means of a motorised shutter in the optical path of the pump pulse. These spectra are obtained by photoexciting the sample using the same TOPAS output as in the gas-phase: $\lambda_{\text {pump }}=290 \mathrm{~nm}$ or $\lambda_{\text {pump }}=308 \mathrm{~nm}$ pump pulses with fluences of $\sim 1 \mathrm{~mJ} \mathrm{~cm}^{-2}$. The probe pulses are derived from a broadband white light continuum (325-675 nm), generated by focusing part of the third $800 \mathrm{~nm}$ fundamental beam $(<5 \%$ of the $1 \mathrm{~mJ}$ per pulse) onto a $1 \mathrm{~mm} \mathrm{CaF}_{2}$ window. The probe pulse polarisation is once again held at the magic angle $\left(54.7^{\circ}\right)$ relative to the pump polarisation, through the use of a half wave plate. The time delay between the pump and probe $(\Delta t)$ is controlled using a second gold retroreflector, mounted on a delay stage situated along the probe beam-path. The present setup provides a temporal resolution of $\sim 80 \mathrm{fs}$ (see $\mathrm{ESI} \dagger$ ).

All transient absorption spectra (TAS) are chirp corrected using the KOALA package ${ }^{36}$ and reported lifetimes are determined using global fitting as previously reported ${ }^{13,37}$ with uncertainties reported to a 95\% confidence interval $(2 \sigma)$ through support plane analysis $^{13,38}$ (see ESI $\dagger$ for further details).

\subsection{Steady state spectroscopic techniques}

Steady state UV-visible spectroscopic measurements were performed on $\sim \mu \mathrm{M}$ solutions of $E$-MMC and $E$-EHMC both in cyclohexane and methanol, in a $1 \mathrm{~cm}$ path length quartz cuvette. The absorption spectra were recorded using a Cary 60 spectrophotometer, see Fig. 1. Difference spectra were recorded using a Cary 300 UV-visible spectrophotometer using the following procedure. For each 'difference spectrum' a steady state UV-visible spectrum of the non-irradiated molecule of interest is subtracted from the UV-visible spectrum obtained following 10 minutes of irradiation $(\sim 3 \mathrm{~W})$. The irradiation is performed with an arc lamp (OBB, tunable KiloArc) providing continuous irradiation at the chosen wavelength. The wavelengths chosen are the same as in the fs pump-probe studies (i.e. $290 \mathrm{~nm}$ in cyclohexane and $308 \mathrm{~nm}$ in methanol).

${ }^{1} \mathrm{H}$ NMR spectra were recorded on a Bruker DPX-300, DPX-400, or AV-250 spectrometer ( $400 \mathrm{MHz}$ for $E$-MMC and $300 \mathrm{MHz}$ for $E$-EHMC) at room temperature in $\mathrm{CDCl}_{3}$. Chemical shifts are given in ppm downfield from the internal standard tetramethylsilane. For the NMR measurements of the irradiated samples, the solution of interest $(\sim 5 \mathrm{mM})$ was placed in a quartz cuvette and irradiated at the maximum of absorption $(290 \mathrm{~nm}$ in cyclohexane and $308 \mathrm{~nm}$ in methanol) for 1 hour (to ensure sufficient quantities of $Z$ isomer had been produced for detection via NMR) with the same apparatus as the difference spectra. The solvents were subsequently removed in vacuo and the collected samples were re-dissolved in $\mathrm{CDCl}_{3}$ before recording the NMR spectra.

\section{Results and discussion}

\subsection{Gas-phase}

Fig. 2 presents TR-IY transients of $E$-MMC ${ }^{+}$and $E$-EHMC ${ }^{+}$ (squares), along with a multiexponential kinetic fit (red trace). Fig. $2 \mathrm{a}$ and $\mathrm{b}$ correspond to TR-IY transients following excitation of $E$-MMC to the $1^{1} \pi \pi^{*}$ state with a $308 \mathrm{~nm}$ and $290 \mathrm{~nm}$ pump respectively, followed by photoionisation with a $200 \mathrm{~nm}$ probe. Both transients are fit with three exponential decay functions, at positive time delays. Cursory inspection shows both transients having a reproducibly small absorption offset that persists out to the maximum time delay of our experiments (900 ps). This baseline offset is modelled in the kinetic fit as an exponential decay with a time-constant, $\tau_{3} \gg 1 \mathrm{~ns}$. We propose that this time-constant corresponds to the relaxation of the long-lived $1^{1} \mathrm{n} \pi^{*}$ state, in accordance with studies by Tan et al. who measured a time-constant of $24 \mathrm{~ns}$ for the $1^{1} \mathrm{n} \pi^{*}$ state decay. ${ }^{22}$ Thus we lock the value to $24 \mathrm{~ns}$ in our three-component kinetic fit, which returns time-constants of $\tau_{1}=1.1 \pm 0.3 \mathrm{ps}$ (at both 308 and $290 \mathrm{~nm}$ pump), $\tau_{2}\left(\lambda_{\text {pump }}=308 \mathrm{~nm}\right)=8 \pm 2 \mathrm{ps}$ and $\tau_{2}\left(\lambda_{\text {pump }}=\right.$ $290 \mathrm{~nm})=5 \pm 3$ ps. Attempts to fit these data with fewer timeconstants produced poorer fits, examples of which may be found in the ESI. $\dagger$

We attempt to reconcile the excited state dynamics of $E$-MMC with reference to the schematic potential energy cuts (PECs) of $E$-MMC shown in Fig. 3 (adapted from ref. 25), alongside the experimental data by Tan et al. as already discussed. ${ }^{22}$ According to the theoretical studies, $E$-MMC undergoes IC from the initially excited $1^{1} \pi \pi^{*}$ state to the $1^{1} n \pi^{*}$ state, facilitated through a $1^{1} \pi \pi^{*} / 1^{1} \mathrm{n} \pi^{*}$ CI. Given that two time-constants are extracted from our fit, we assign $\tau_{1}$ to intramolecular vibrational relaxation (IVR) within the $1^{1} \pi \pi^{*}$ state, followed by IC via $1^{1} \pi \pi^{*} \rightarrow 1^{1} n \pi^{*}$ (see Fig. 3), represented by $\tau_{2}$. The energy dependence of $\tau_{2}$ would then likely be a reflection of the increased vibrational energy which facilitates the IC through the CI. Taken together, $\tau_{1}$ and $\tau_{2}$ correspond to the evolution of $E$-MMC towards the $1^{1} \mathrm{n} \pi^{*}$ state which subsequently decays to $S_{0}$ with a time-constant of $\tau_{3}=24 \mathrm{~ns}^{22} \mathrm{We}$ note that due to the complexity of the molecules under study it is not possible to unambiguously assign individual processes to single time constants. However, similar time constants have been assigned to an IVR/IC process in the much simpler molecule, $N$-methylpyrrole, ${ }^{39}$ which guide our assignments here.

Two caveats are in order here. Firstly, we recognise that within our spectral bandwidth, we are very likely exciting multiple rotamers in our molecular beam. ${ }^{22,26}$ Indeed, measurements performed by Tan et al. suggest that the dynamics are faster for s-trans-E-MMC (rotamer with the highest excitation energy) than for s-cis-E-MMC (rotamer with the lowest excitation energy), separated by $\sim 340 \mathrm{~cm}^{-1}$ at their vibronic origins (see ESI $\dagger$ for structures). It is possible therefore that the different values for $\tau_{2}$ returned here are due to the different extent of rotamer excitation in our molecular beam although we recognise that the values of 

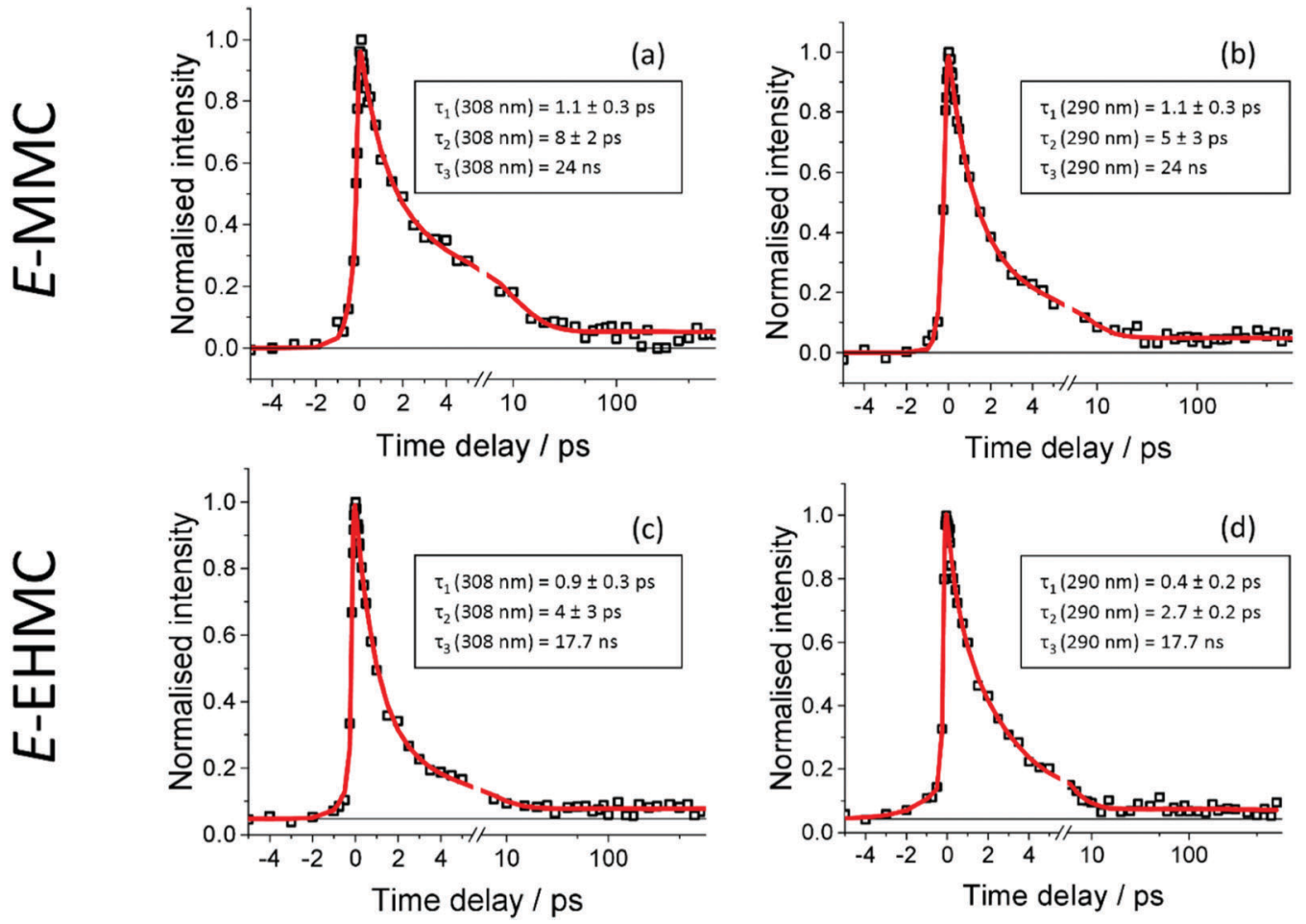

Fig. 2 (a) and (b) TR-IY signal (black squares) of $E$-MMC with $\lambda_{\text {pump }}=290 \mathrm{~nm}$ and $\lambda_{\text {pump }}=308 \mathrm{~nm}$ respectively. (c) and (d) The same but with $E$-EHMC. In all cases, the red trace corresponds to a multiexponential fit. The horizontal black line serves as a visual aid for the long lived $n \pi^{\star}$ state.

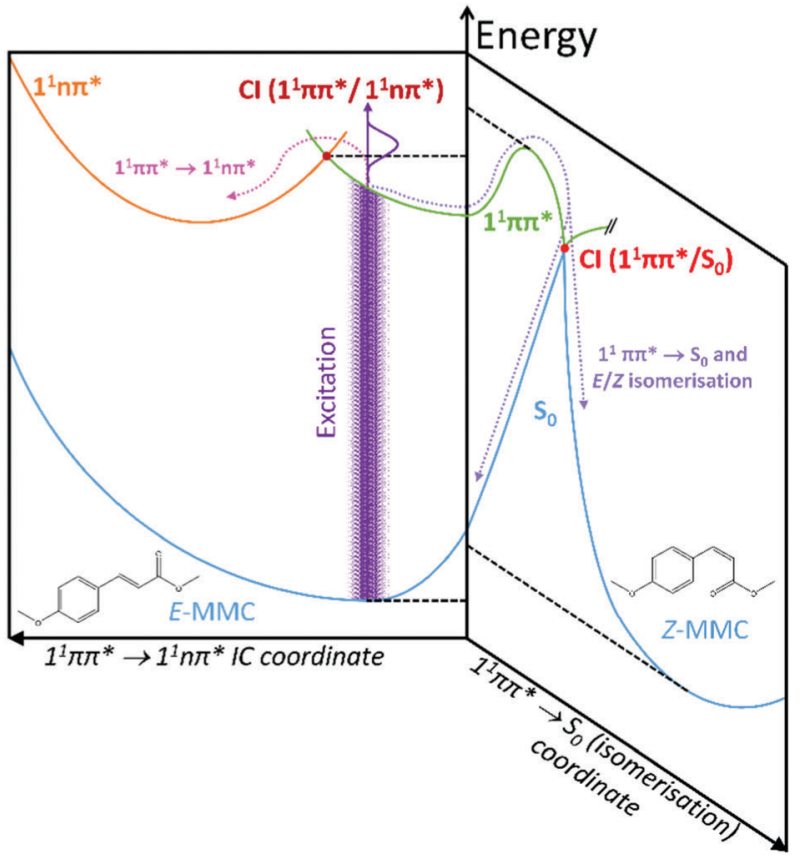

Fig. 3 Schematic cuts of the potential energy surfaces (PESs) showing the two reaction coordinates considered in this study; adapted from ref. 25.

$\tau_{2}$ for each wavelength agree within error. Secondly, the probability of $E / Z$ isomerisation is low, given the energy barrier that is required to be surmounted along the $1^{1} \pi \pi^{*}$ state $\left(\sim 1700 \mathrm{~cm}^{-1},{ }^{25}\right.$ dashed horizontal, see Fig. 3). That said, even if $E / Z$ isomerisation was operative, we would be unable to probe this pathway given that, once population of the $Z$ isomer (or $E$ isomer) in $\mathrm{S}_{0}$ has ensued (via a $1^{1} \pi \pi^{*} / S_{0}$ CI; see Fig. 3), one photon of the $200 \mathrm{~nm}$ probe would have insufficient energy to photoionise out of this state.

Fig. 2c and d correspond to TR-IY transients following excitation of $E$-EHMC to the $1^{1} \pi \pi^{*}$ state with a $308 \mathrm{~nm}$ and $290 \mathrm{~nm}$ pump, respectively, followed by photoionisation with a $200 \mathrm{~nm}$ probe. The same fit function as for $E$-MMC is used to model the data: the value of $\tau_{3}$ is fixed at $17.7 \mathrm{~ns}$, using the value obtained by Tan et al., with the remaining two time-constants determined by the fit being $\tau_{1}\left(\lambda_{\text {pump }}=308 \mathrm{~nm}\right)=0.9 \pm 0.3 \mathrm{ps}, \tau_{1}\left(\lambda_{\text {pump }}=\right.$ $290 \mathrm{~nm})=0.4 \pm 0.2 \mathrm{ps}, \tau_{2}\left(\lambda_{\text {pump }}=308 \mathrm{~nm}\right)=4 \pm 3$ ps and $\tau_{2}$ $\left(\lambda_{\text {pump }}=290 \mathrm{~nm}\right)=2.7 \pm 0.2 \mathrm{ps}$. These values agree with the timeconstants for $E$-MMC, but are slightly shorter, suggesting that $E$-EHMC undergoes the same dynamic processes as $E$-MMC. The long alkyl chain, which inevitably increases the density of vibrational states, may facilitate faster IVR and/or IC. This could, therefore, reconcile the slight increase in relaxation rates in going from $E$-MMC to $E$-EMHC.

We draw discussion of our gas-phase data to a close, by considering the most recent ps studies by Miyazaki et al. on $E$-MMC. ${ }^{26}$ Our results strongly suggest that the excited state dynamics of $E$-MMC can be modelled with three timeconstants. Two of these time-constants are $<10 \mathrm{ps}$ and one $\gg$ ns. In contrast, Miyazaki et al. report an energy-dependence in the excited state dynamics of $E$-MMC, with extracted timeconstants ranging from 13 to 280 ps depending on which rotamer (and vibronic state therein) was photoexcited. For completeness, 
we note that the 12 ps temporal resolution used by Miyazaki et al. would not allow observation of the few ps time-constants presented above. The present measurements utilise pump pulses with a spectral bandwidth of $\sim 500 \mathrm{~cm}^{-1}$, meaning that we are exciting all rotamers (together with any vibronic manifolds) simultaneously. We should then anticipate, perhaps, a convolution of the time-constants observed by Miyazaki et al. However, the time constants observed are either much shorter (1.1-8 ps) or much longer ( $\gg \mathrm{ns}$ ) than would be expected for such a convolution. We note that Miyazaki et al. ${ }^{40}$ used a $315 \mathrm{~nm}$ probe, with picosecond time-resolution, whereas to ensure ionisation out of both $1^{1} \pi \pi^{*}$ and $1^{1} \mathrm{n} \pi^{*}$ states, a $200 \mathrm{~nm}$ probe was used in the present work. This will inevitably lead to differences in Franck-Condon factors out of these states, as compared to using a $315 \mathrm{~nm}$ probe. While the results of Miyazaki et al. could not be replicated with a $200 \mathrm{~nm}$ probe, we tentatively suggest that we do observe the expected convolution of time-constants when probing at $315 \mathrm{~nm}$, where we see a component with a lifetime in the hundreds of picoseconds (see $\mathrm{ESI} \dagger$ ). The fact that we do see this component when probing at $315 \mathrm{~nm}$ and do not at $200 \mathrm{~nm}$, emphasises that the discrepancies between our results and those of Miyazaki et al. are likely the result of the difference in probe wavelength rather than the difference in temporal resolution.
Importantly, in the present studies, new insight has been garnered into the initial photophysics and photochemistry of the sunscreen agent E-EHMC in the gas-phase.

\subsection{Solution-phase}

We first consider the TAS obtained in E-MMC following photoexcitation at $290 \mathrm{~nm}$ in cyclohexane and $308 \mathrm{~nm}$ photoexcitation in methanol, depicted in Fig. 4a and b respectively. We specifically chose these two solvents to reflect the effects, upon the excited state dynamics of $E$-MMC, of both a weakly perturbing and strongly perturbing (hydrogen bonding) solvent, respectively. Given the differences in excited state potential energies ${ }^{25}$ and static absorption spectra (as discussed in Section 1), we would expect a qualitative difference in the TAS features, induced by solvent environment. Following population of the $1^{1} \pi \pi^{*}$ state, both TAS show a large excited state absorption (ESA) centred at $400 \mathrm{~nm}$ for $E$-MMC in cyclohexane and $380 \mathrm{~nm}$ for $E$-MMC in methanol. The TAS for methanol solution also show a second feature centred at $\sim 360 \mathrm{~nm}$ (probe wavelength), which is present after the decay of the $380 \mathrm{~nm}$ feature. Similarly, close examination of the cyclohexane TAS shows a small absorption increase at $<330 \mathrm{~nm}$ (more obvious in the E-EHMC TAS in Fig. 4e) appearing after the initial decay of the $400 \mathrm{~nm}$ peak, and decaying in a few picoseconds. Both TAS almost recover to zero within 5 ps,
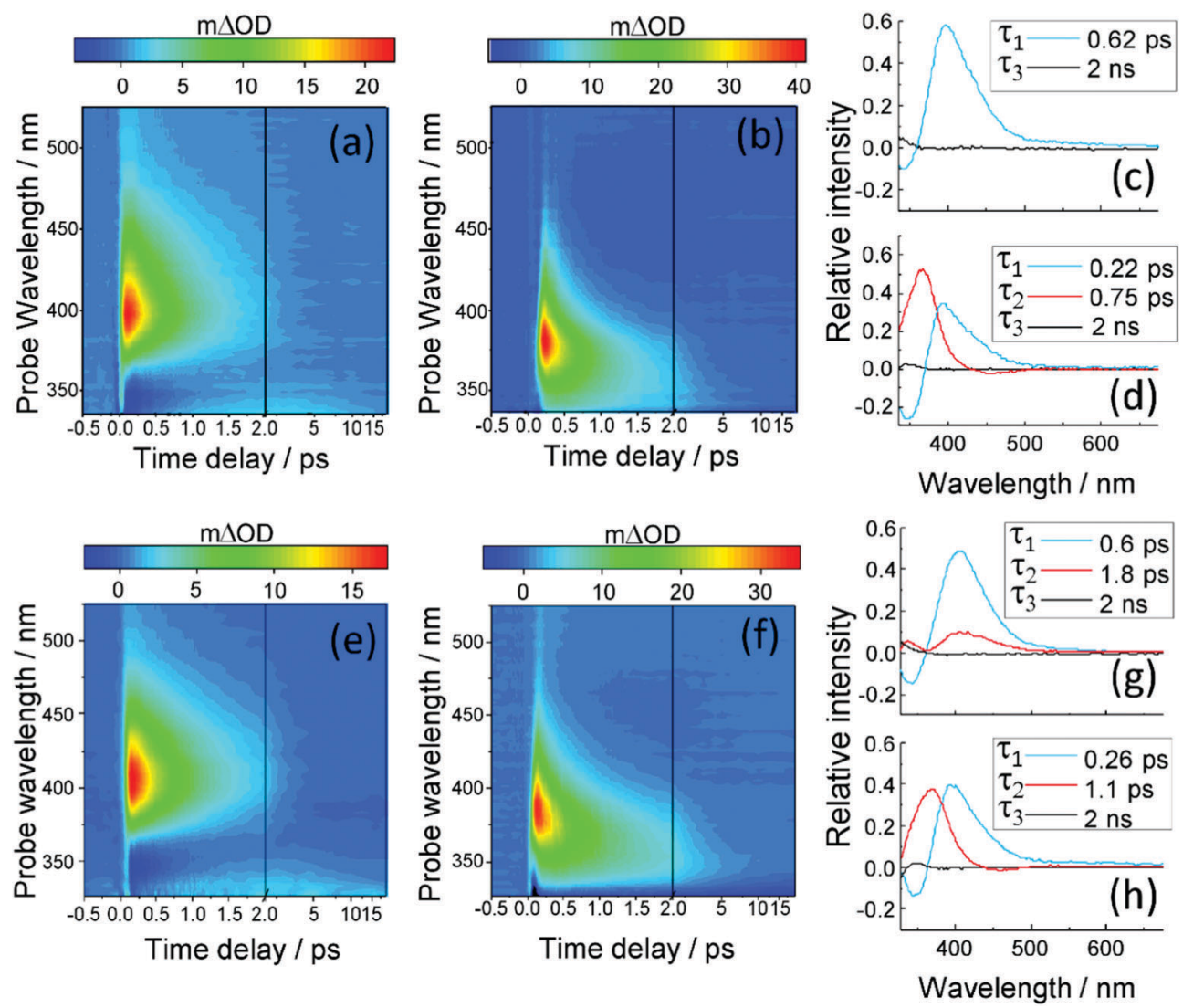

Fig. 4 (a) and (b) TAS of E-MMC in cyclohexane $\left(\lambda_{\text {pump }}=290 \mathrm{~nm}\right)$ and methanol $\left(\lambda_{\text {pump }}=308 \mathrm{~nm}\right)$. (c) and (d) DAS corresponding to (a) and (b) respectively. (e) and (f) TAS of E-EHMC in cyclohexane $\left(\lambda_{\text {pump }}=290 \mathrm{~nm}\right)$ and methanol $\left(\lambda_{\text {pump }}=308 \mathrm{~nm}\right)$. (g) and (h) DAS corresponding to (e) and (f) respectively. 

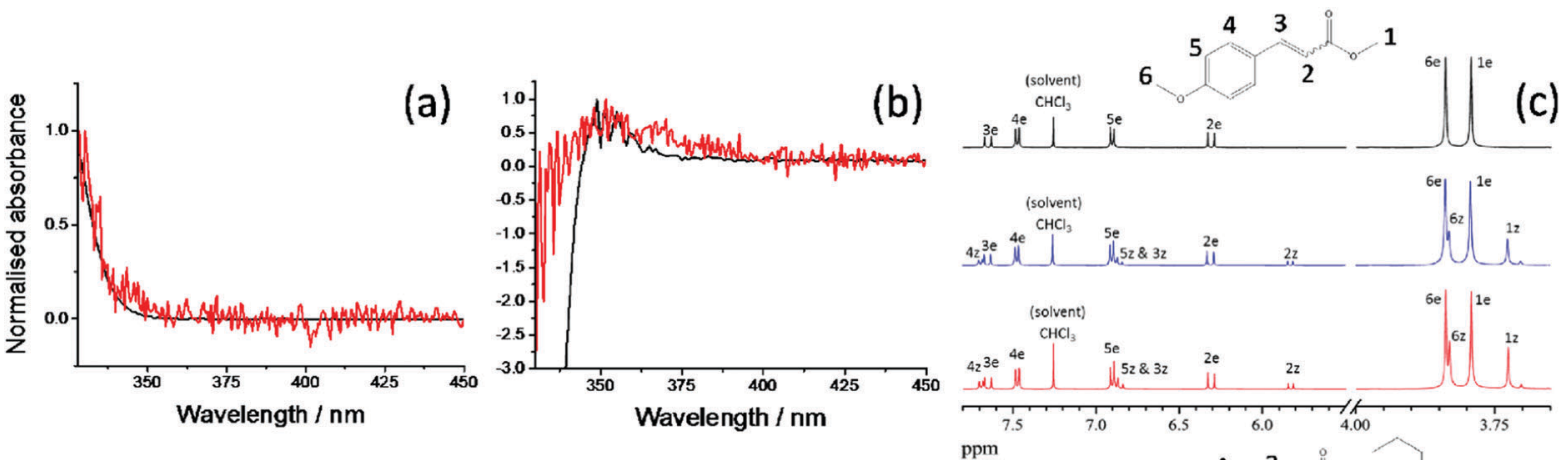

(e)
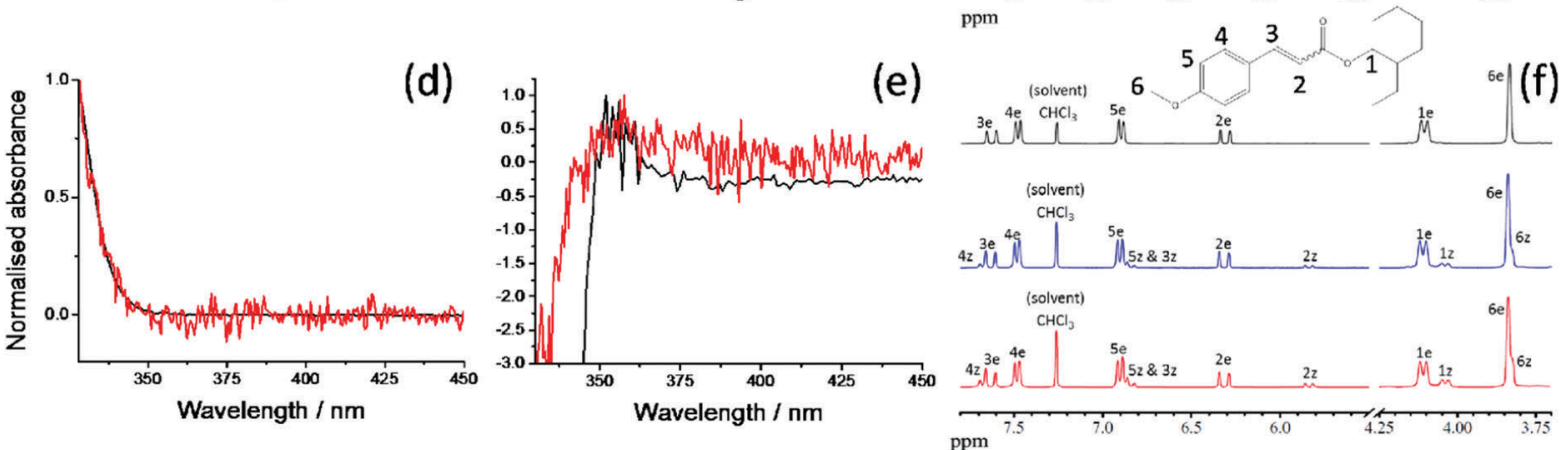

Fig. 5 Normalised difference spectra (black traces) for $E-M M C$ in (a) cyclohexane $\left(\lambda_{\text {pump }}=290 \mathrm{~nm}\right)$ and (b) methanol $\left(\lambda_{\text {pump }}=308 \mathrm{~nm}\right) \mathrm{compared}$ with the corresponding TAS (red traces) at $2 \mathrm{~ns}$; (c) ${ }^{1} \mathrm{H}$ NMR spectra $\left(\mathrm{CDCl}_{3}, 400 \mathrm{MHz}\right)$ of $E-M M C$ and peak attribution using the proton numbering presented inset and labelling the protons from the $E$ and $Z$ isomers as ' $e$ ' and ' $z$ ' respectively: before irradiation (black trace), after irradiation at $290 \mathrm{~nm}$ for 1 hour in cyclohexane (blue trace) and after irradiation at $308 \mathrm{~nm}$ for 1 hour in methanol (red trace). (d) - (f) Similar but for $E$-EHMC and ${ }^{1} \mathrm{H} ~ N M R$ spectra (CDCl $3,300 \mathrm{MHz}$ ). Everything below 3.7 ppm has been removed for clarity (see ESI $\dagger$ for detailed spectra).

indicative of very fast repopulation of $\mathrm{S}_{0}$. Fig. $5 \mathrm{a}$ and $\mathrm{b}$ show the TAS acquired at $2 \mathrm{~ns}$ (red trace), showing a positive feature at $<340 \mathrm{~nm}$ in cyclohexane and $350 \mathrm{~nm}$ in methanol. For $E$-MMC in methanol, there is also a large negative signal at wavelengths shorter than $350 \mathrm{~nm}$ (where $E$-MMC absorbs, see Fig. 1). As we will discuss shortly, these spectra show that $S_{0}$ recovery is incomplete within our temporal window, a signifier of either a long-lived intermediary species or photoproduct.

The TAS were globally fit in order to extract the time-constants related to the different dynamical behaviours of $E$-MMC in the two solvents. The decay associated spectra (DAS) corresponding to the TAS shown in Fig. $4 \mathrm{a}$ and b, are presented in Fig. 4c and d, respectively. With such DAS, a positive amplitude corresponds to a decay in the TAS signal, a negative amplitude to a growth.

Table 1 Time-constants extracted from the global fitting of solutionphase TAS, after $290 \mathrm{~nm}$ and $308 \mathrm{~nm}$ excitation, in cyclohexane and methanol respectively, both for $E-M M C$ and for $E$-EHMC

\begin{tabular}{lllll}
\hline Compound; solvent & Wavelength & $\tau_{1} / \mathrm{ps}$ & $\tau_{2} / \mathrm{ps}$ & $\tau_{3} / \mathrm{ns}$ \\
\hline$E$-MMC; cyclohexane & $\lambda_{\text {pump }}=290 \mathrm{~nm}$ & $0.62 \pm 0.02$ & $\sim 3^{a}$ & $>2.0$ \\
-MMC; methanol & $\lambda_{\text {pump }}=308 \mathrm{~nm}$ & $0.22 \pm 0.04$ & $0.75 \pm 0.09$ & $>2.0$ \\
$E$-EHMC; cyclohexane & $\lambda_{\text {pump }}=290 \mathrm{~nm}$ & $0.6 \pm 0.1$ & $1.8 \pm 1.2^{b}$ & $>2.0$ \\
$E$-EHMC; methanol & $\lambda_{\text {pump }}=308 \mathrm{~nm}$ & $0.26 \pm 0.09$ & $1.1 \pm 0.3$ & $>2.0$
\end{tabular}

${ }^{a}$ A weak feature with a $\sim 3$ ps decay is observed in the TAS around $330 \mathrm{~nm}$. However, it was not possible to produce an accurate fit for it because of its low intensity and its position on the edge of our probe window. ${ }^{b}$ Contrary to $E$-MMC in cyclohexane, $\tau_{2}$ is needed to have a good fit, but the uncertainty is large, see ESI.
As reported in Table 1, three time-constants are required to account for the excited state dynamics of $E$-MMC in cyclohexane and methanol. The first two time-constants are hundreds of fs in duration each, the fastest of which $\left(\tau_{1}\right)$ shows a positive amplitude in the DAS at $\sim 400 \mathrm{~nm}$ and negative signal further to blue, usually indicative of population flow between two states. The middle time-constant $\left(\tau_{2}\right)$, where relevant, shows principally a positive DAS amplitude. The final time-constant is assigned as $\tau_{3}>2 \mathrm{~ns}$, used to fit a long-lived component in the TAS (i.e. a new species which lives beyond our temporal window), and manifests in the DAS as a small positive feature at $\sim 340 \mathrm{~nm}$. Cursory inspection of the DAS in Fig. 4 and time-constants reported in Table 1 show notable differences upon changing solvent, suggesting that the hydrogen bonding in methanol may have some effect on the relaxation dynamics of $E$-MMC. An additional timeconstant, not included in the data presented in Table 1, was necessary to produce a good fit in both solvents. This component, which is below the temporal resolution of our experiment, is assigned to the response from solvent- and $\mathrm{CaF}_{2}$ windowdynamics from the Harrick Cell (see ESI $\dagger$ ) and, therefore, will not be discussed further.

We begin our analysis by addressing the long lived species $\left(\tau_{3}\right)$ present in both cyclohexane and methanol. Based on previous experimental and theoretical studies on $E$-MMC ${ }^{24,25}$ and related molecules, ${ }^{15,19,41}$ there is sufficient precedent that $E$-MMC is likely to form Z-MMC, or other photoproducts, upon photoexcitation. To verify this, we recorded difference spectra (see Section 2.3) of E-MMC in both cyclohexane and methanol, following irradiation 
at the maximum spectral absorbance (see Fig. 1); $290 \mathrm{~nm}$ in cyclohexane and $308 \mathrm{~nm}$ in methanol. These are shown by the black traces given in Fig. 5a and b for cyclohexane and methanol, respectively. As with the 2 ns TAS, we can see that there is a clear positive feature at $<340 \mathrm{~nm}$ in cyclohexane and at $\sim 350 \mathrm{~nm}$ in methanol. These results suggest that after 10 minutes of irradiation, there is a drop in absorption from the initial molecule, along with the emergence of a new species absorbing at $<340 \mathrm{~nm}$ in cyclohexane and at $\sim 350 \mathrm{~nm}$ in methanol. Moreover, the shape of the difference spectra in cyclohexane (more noticeably) and methanol, accord nicely with the TAS at 2 ns (red traces in Fig. 5a and b) indicating that the new absorbing species are formed in less than $2 \mathrm{~ns}$ and have extended lifetimes (i.e. are chemically stable). Theoretical calculations ${ }^{25}$ (see Fig. 3) and a previous study of $E$-MMC embedded in neon matrices, ${ }^{24}$ suggest that the observed photoproduct is the $Z$-MMC isomer. The reduced correlation between the 2 ns TAS and the difference spectrum in methanol is likely due to the appearance of another, less stable, photoproduct, which is most likely attributed to a phenolic radical species. This radical is formed via ionisation to generate solvated "free"-electrons, ${ }^{10,20,43,44}$ as has been previously observed in similar cinnamate-based systems. ${ }^{10,20}$ Indeed the TAS in methanol at $2 \mathrm{~ns}$ seems to present a small absorption feature around $600 \mathrm{~nm}$ which is not observed in the difference spectrum (see $\mathrm{ESI} \dagger$ ). We tentatively suggest that this feature corresponds to solvated electrons ${ }^{42}$ formed by multiphoton ionisation of $E$-MMC to produce the unstable radical cation $E-\mathrm{MMC}^{+}$(which subsequently generates the phenolic radical species), ${ }^{10,20,43,44}$ although the poor signal-to-noise in the data makes definitive assignment impossible. In any case, in a sunscreen context, the formation of this photoproduct is of little importance as multiphoton absorption is unlikely to occur under sun light irradiance. In both the 2 ns TAS and the difference spectrum, there appears to be a positive offset, the origins of which are not clear. It is worth noting, however, that signal levels were particularly low in this region $(\sim 10 \mu \Delta \mathrm{OD})$, well within the error of the instruments involved, and so any quantitative analysis in this region is unfeasible.

In order to further validate the $E / Z$ photoisomerisation pathway in both the dynamic and steady-state measurements presented in Fig. 4 and 5 respectively, we recorded ${ }^{1} \mathrm{H}$ NMR spectra of samples containing E-MMC in both cyclohexane and methanol, pre- and post-irradiation with the wavelength corresponding to the maximum of absorption in the two different solvents (see Fig. 1). The NMR spectra obtained are shown in Fig. 5c. Detailed spectra and further explanations are provided in the ESI. $\dagger$ The non-irradiated NMR spectrum (top) shows that the starting material is the $E$ isomer exclusively. Upon irradiation, $Z$ isomer is clearly present in the sample, as shown by the appearance of the new peaks in the NMR spectra postirradiation (middle and bottom). By integrating the different peaks, we are able to estimate the relative proportions of $E$ and $Z$ isomers after one hour of irradiation, which gives $\sim 22 \%$ of $Z$ isomer in cyclohexane and $\sim 28 \%$ in methanol. Even though we may not have reached the $E / Z$ equilibrium ratio after only onehour irradiation, the $E / Z$ isomerisation seems to be slightly more efficient in methanol than in cyclohexane and the values are in good agreement with previous quantitative observations on photoisomerisation of EHMC. ${ }^{45}$

Given the evidence presented above, it is clear that photoexcited $E$-MMC rapidly returns to the ground electronic state as either the $E$ or $Z$ isomer. With this assignment, we now attempt to interpret $E$-MMC's short time behaviour in the solution-phase following UV-B photoexcitation to the $1^{1} \pi \pi^{*}$ state, particularly in light of the PECs schematised in Fig. 3. We first note, however, that $E$-MMC undergoes numerous processes that are likely convoluted together, thus making distinct assignment of any one time-constant with a molecular process difficult. We propose the following process for $E$-MMC both in cyclohexane and methanol: following photoexcitation to the $1^{1} \pi \pi^{*}$ state, $E$-MMC undergoes IVR $\left(\tau_{1}\right)$, as evidenced by the rapid drop in intensity of the $\sim 400 \mathrm{~nm}$ feature in the TAS and the corresponding rise at $<375 \mathrm{~nm}$. This is characterised by the blue traces in the DAS in Fig. 4. Qualitatively, we observe this as a shift in intensity from $\sim 380 \mathrm{~nm}$ to $\sim 360 \mathrm{~nm}$ in the methanol TAS. Unfortunately, while we believe a similar process is occurring in cyclohexane, this intensity shift is less obvious: at wavelengths shorter than $400 \mathrm{~nm}$ the only positive feature appears at $<330 \mathrm{~nm}$. The apparently flat cyclohexane TAS in this region ( $c a .350 \mathrm{~nm}$ and $500 \mathrm{fs}$ ) may be attributed to overlapping positive excited state absorption and negative ground state bleach (and/or stimulated emission) features. The spectral shift of these features between solvents is likely due to the relative stability of the ${ }^{1} \pi \pi^{*}$ states in methanol as compared to cyclohexane (as indicated in the static spectra in Fig. 1).

Subsequent to this initial relaxation, the excited state population evolves along the $E / Z$ isomerisation coordinate, likely along with any solvent rearrangement that may occur, leading to $1^{1} \pi \pi^{*} \rightarrow S_{0}$ IC facilitated through the corresponding $1^{1} \pi \pi^{*} / \mathrm{S}_{0} \mathrm{CI}$ (convoluted in $\tau_{2}$ ). This latter step accords with the mixture of $E / Z$ isomers, given bifurcation of excited state population into two product channels (Fig. 3). In $E$-MMC in methanol, the positive DAS feature centred at $360 \mathrm{~nm}$ supports this assignment. Unfortunately, due to a low signal-to-noise ratio (likely due to competition between excited state absorption and ground state bleach/stimulated emission, vide supra), we were unable to fit the TAS feature at $<330 \mathrm{~nm}$ in $E$-MMC in cyclohexane. We have, however, estimated the timeconstant as a few picoseconds by visual inspection (see Table 1).

Moreover, it is worth noting that the dynamics are, in general (see also for $E$-EHMC), shorter in methanol than in cyclohexane. As discussed, this may be due to the lowering of the $1^{1} \pi \pi^{*} \rightarrow S_{0}$ transition barrier, predicted by $a b$ initio calculations of $E$-MMC with a single, hydrogen bonded solvent molecule, ${ }^{25}$ and the wellknown destabilisation of the $1^{1} n \pi^{*}$ state in a polar-protic solvent, ${ }^{23}$ which ultimately suppresses the $1^{1} \pi \pi^{*} \rightarrow 1^{1} \mathrm{n} \pi^{*}$ transition. Interestingly, the still-anticipated low $1^{1} \pi \pi^{*} \rightarrow 1^{1} n \pi^{*}$ barrier in cyclohexane (assuming very little perturbation to these states relative to the gas-phase) cannot prevent this transition from happening. However, gas-phase data show that the $1^{1} n \pi^{*}$ state is a long-lived state and it is likely that, in the solution phase (given the thermal energy at room temperature in solution), population exists in, and can flow between, both the $1^{1} \pi \pi^{*}$ and the $1^{1} n \pi^{*}$ until the $1^{1} \pi \pi^{*} / S_{0}$ CI is accessed. ${ }^{25}$ The global reaction seems 
likely, thus, to be led by the $1^{1} \pi \pi^{*} \rightarrow S_{0}$ transition, leading to efficient population from all the excited molecules to $S_{0}$ in either $E$ or $Z$ isomeric forms.

In addition to $E$-MMC, identical studies have been performed on $E$-EHMC: the results are presented in Fig. $4 \mathrm{e}$ and $\mathrm{f}$ for the TAS; Fig. $4 \mathrm{~g}$ and $\mathrm{h}$ for the corresponding DAS; Fig. $5 \mathrm{~d}$ and e for the difference spectra, Fig. $5 \mathrm{f}$ for the NMR study; and in Table 1 for the time-constants extracted from the global fits. Taken together, the results are almost commensurate to $E$-MMC's, with two main differences. Firstly, even though the values of $\tau_{2}$ overlap within error, we note that $\tau_{1}$ and $\tau_{2}$ are globally greater for $E$-EHMC than for $E$-MMC. That may be linked to the lengthening of the alkyl chain, which may provide some inertia towards rotation about the $\mathrm{C}=\mathrm{C}$ bond. ${ }^{46}$ We also note here that the limitation of the global fitting procedure means that all the lifetimes correspond to processes that start from time zero. As such, one expects to have 'blurring' of one dynamical process with the other (particularly when commensurate), and hence the effect on both $\tau_{1}$ and $\tau_{2}$. Secondly, the NMR spectra suggest an $E / Z$ isomerisation leading to $\sim 15 \%$ and $\sim 23 \%$ of $Z$ isomer in cyclohexane and methanol, respectively. This suggests that $E$-EHMC undergoes similar $E / Z$ photoisomerisation dynamics as $E$-MMC, albeit with subtle differences. Moreover, this mechanism, along with the timeconstants obtained, is in agreement with the fluorescence study of $E$-EHMC, both in hexane and methanol, which infers fluorescence lifetimes of less than 10 ps. $^{45,47}$

We close by noting one final caveat. An alternative explanation for the attribution of the time-constants in both $E$-MMC and $E$-EHMC should be considered: $\tau_{1}$ could correspond to the convolution of IVR, the evolution along the $E / Z$ isomerisation coordinate on the PES, and the $1^{1} \pi \pi^{*} \rightarrow S_{0}$ de-excitation through the corresponding CI. $\tau_{2}$ would then model the cooling of vibrationally hot MMC or EHMC in the $E / Z$ ground state, as has been observed in other systems. ${ }^{35,48}$ At this point we are unable to conclusively propose one mechanism over the other. However, this is considered unlikely in methanol solution as the slight negative component in the TAS in Fig. $4 \mathrm{~b}$ and $\mathrm{f}$ at $\sim 450 \mathrm{~nm}$ and $\sim 2 \mathrm{ps}$ time delay (see ESI $\dagger$ ), is indicative of stimulated emission (also evidenced in the associated DAS in Fig. $4 \mathrm{~d}$ and $\mathrm{h}$ at the same wavelength) which will not occur from a vibrationally hot ground state. While the same stimulated emission is not observed in cyclohexane, the similarity in timeconstants observed between the two solvents make it likely that the same processes are occurring in each. As such, while the above caveat cannot be entirely ruled out, we suggest it to be less likely than the arguments presented in this work (vide supra). Importantly, however, this work reports the first combined investigation on $E$-MMC and E-EHMC's ultrafast behaviour in solution, and gives possible explanations for the ultrafast dynamics in both polar-protic and non-polar-aprotic solvents.

\section{Conclusions}

The present study reports on the excited state dynamics of one of the leading sunscreen filter molecules used in off-the-shelf sunscreens, $E$-EHMC, in both the gas- and solution-phase, along with its model chromophore, E-MMC. Both of these molecules display seemingly similar dynamics.

In the gas-phase, photoexcitation to the $1^{1} \pi \pi^{*}$ state leads to a deactivation process characterised by three time-constants: $\tau_{1}, \tau_{2}$ and $\tau_{3} . \tau_{1}$, which ranges from $\sim 0.4 \mathrm{ps}$ to $\sim 1.1 \mathrm{ps}$, is dependent on both the excitation energy in the $1^{1} \pi \pi^{*}$ state and the molecule studied ( $E$-MMC or $E$-EHMC). $\tau_{2}$ ranges between $\sim 2.7$ and $\sim 8 \mathrm{ps}$ and is dependent on similar parameters as $\tau_{1}$. Finally, $\tau_{3}$ extends beyond the time-window of our measurements ( $\gg 0.9 \mathrm{~ns}$ ). Our findings, guided closely by previous studies, lead us to suggest that $\tau_{1}$ and $\tau_{2}$ correspond to intramolecular vibrational relaxation within the $1^{1} \pi \pi^{*}$ state and non-radiative decay, via internal conversion, from the $1^{1} \pi \pi^{*}$ to the $1^{1} n \pi^{*}$ state, respectively. $\tau_{3}$ then models the de-excitation from the $1^{1} n \pi^{*}$ state to $S_{0}$. The dependence on energy of $\tau_{1}$ and $\tau_{2}$ can be rationalised assuming a barrier along the reaction coordinate and/or different rotamers excited within our molecular beam. However, additional theoretical calculations would be helpful to confirm our results, such as the determination of the barriers along the $1^{1} \pi \pi^{*} \rightarrow 1^{1} n \pi^{*}$ and the $1^{1} \pi \pi^{*} \rightarrow \mathrm{S}_{0}$ coordinates for the $s$-trans rotamer, which would confirm the energy dependence of $\tau_{2}$.

Using both a non-polar-aprotic solvent, cyclohexane, and a polar-protic solvent, methanol, the photochemistry of $E$-MMC and $E$-EHMC in a closer-to-realistic environment shows noticeable differences to the gas-phase. Whilst the deactivation processes are equally fast, requiring three time-constants to model, comparative steady state UV and ${ }^{1} \mathrm{H}$ NMR data suggest that the long-lived component characterised by $\tau_{3}$ is attributable to the $Z$-MMC and Z-EHMC photoproducts and not population trapped in the $1^{1} n \pi^{*}$ state ( $c f$. gas-phase). This leads us to propose that excited state deactivation involves flow of population from the vertical Franck-Condon region induced by intramolecular vibrational relaxation. This is characterised by $\tau_{1} \cdot \tau_{2}$ then likely corresponds to excited state population evolving along the isomerisation coordinate along with internal conversion from $1^{1} \pi \pi^{*}$ to $S_{0}$, mediated by an appropriate $1^{1} \pi \pi^{*} / S_{0}$ conical intersection, along with any associated solvent rearrangement. $\tau_{3}$ then reflects the long-lived $Z$-EHMC photoproduct. However, further investigation is needed, especially pump-probe measurements utilising a UV pump and IR probe, which would allow the direct observation of IVR, as well as the $E / Z$ isomerisation, and validate the attribution of $\tau_{2}$ by seeking to observe vibrational cooling in $\mathrm{S}_{0}$.

As a final comment, $E$-MMC is a good basis for the development of future sunscreens like E-EHMC. The present study shows that $E$-MMC and $E$-EHMC do not produce any photofragments within $2 \mathrm{~ns}$, following excitation at $308 \mathrm{~nm}$ and $290 \mathrm{~nm}$, apart from radical species formed via multiphoton absorption, which is unlikely to occur with the photon flux from sun light irradiance. In fact, all the excited molecules return to $S_{0}$ as one of the two possible stereoisomers, $E$ or $Z$. Moreover, de-excitation to the ground state is ultrafast, efficiently removing potentially harmful excited $E$-EHMC from the skin. The reported results highlight the power and sensitivity of pump-probe spectroscopic techniques as well as the use of a 'double' bottom-up approach (complementary gas-phase and solution-phase studies alongside an increase in 
molecular complexity) to understand the photoprotection pathways of sunscreen agents in general, and E-EHMC's behaviour in particular.

\section{Acknowledgements}

Y. P. thanks the Ecole Normale Supérieure (ENS) de Cachan and French state for financing his studies. M. S. and N. D. N. R. thank the Engineering and Physical Sciences Research Council (EPSRC) for postdoctoral and doctoral funding respectively. L. A. B. and W. D. Q. thank the EPSRC for providing studentships under grant EP/F500378/1, through the Molecular Organisation and Assembly in Cells Doctoral Training Centre. N. C. C. F. thanks the Leverhulme Trust for postdoctoral funding. V. G. S. thanks the EPSRC for an equipment grant (EP/J007153) and the Royal Society for a University Research Fellowship. The authors thank Drs Jamie Young and Michael Horbury (University of Warwick) for their technical assistance and for helpful comments when preparing the manuscript.

\section{References}

1 C. E. Crespo-Hernández, B. Cohen, P. M. Hare and B. Kohler, Chem. Rev., 2004, 104, 1977.

2 C. T. Middleton, K. de La Harpe, C. Su, Y. K. Law, C. E. Crespo-Hernández and B. Kohler, Annu. Rev. Phys. Chem., 2009, 60, 217.

3 W. J. Schreier, T. E. Schrader, F. O. Koller, P. Gilch, C. E. Crespo-Hernández, V. N. Swaminathan, T. Carell, W. Zinth and B. Kohler, Science, 2007, 315, 625.

4 G. P. Pfeifer, Y. H. You and A. Besaratinia, Mutat. Res., Fundam. Mol. Mech. Mutagen., 2005, 571, 19.

5 W. W. Sprenger, W. D. Hoff, J. P. Armitage and K. J. Hellingwerf, J. Bacteriol., 1993, 175, 3096.

6 K. J. Hellingwerf, J. Hendriks and T. Gensch, J. Phys. Chem. A, 2003, 107, 1082.

7 U. K. Genick, S. M. Soltis, P. Kuhn, I. L. Canestrelli and E. D. Getzoff, Nature, 1998, 392, 206.

8 M. M. Caldwell, J. F. Bornman, C. L. Ballaré, S. D. Flint and G. Kulandaivelu, Photochem. Photobiol. Sci., 2007, 6, 252.

9 G. I. Jenkins, Annu. Rev. Plant Biol., 2009, 60, 407.

10 M. Vengris, D. S. Larsen, M. A. van der Horst, O. F. A. Larsen, K. J. Hellingwerf and R. van Grondelle, J. Phys. Chem. B, 2005, 109, 4197.

11 E. M. M. Tan, S. Amirjalayer, B. H. Bakker and W. J. Buma, Faraday Discuss., 2013, 163, 321.

12 J. C. Dean, R. Kusaka, P. S. Walsh, F. Allais and T. S. Zwier, J. Am. Chem. Soc., 2014, 136, 14780.

13 L. A. Baker, M. D. Horbury, S. E. Greenough, F. Allais, P. S. Walsh, S. Habershon and V. G. Stavros, J. Phys. Chem. Lett., 2016, 7, 56.

14 E. V. Gromov, I. Burghardt, H. Köppel and L. S. Cederbaum, J. Phys. Chem. A, 2005, 109, 4623.

15 T. N. V. Karsili, B. Marchetti, M. N. R. Ashfold and W. Domcke, J. Phys. Chem. A, 2014, 118, 11999.
16 S. Smolarek, A. Vdovin, D. L. Perrier, J. P. Smit, M. Drabbels and W. J. Buma, J. Am. Chem. Soc., 2010, 132, 6315.

17 N. D. N. Rodrigues, M. Staniforth, J. D. Young, Y. Peperstraete, N. C. Cole-Filipiak, J. R. Gord, P. S. Walsh, D. M. Hewett, T. S. Zwier and V. G. Stavros, Faraday Discuss., 2016, DOI: 10.1039/C6FD00079G.

18 M. de Groot, E. V. Gromov, H. Köppel and W. J. Buma, J. Phys. Chem. B, 2008, 112, 4427.

19 D. Shimada, R. Kusaka, Y. Inokuchi, M. Ehara and T. Ebata, Phys. Chem. Chem. Phys., 2012, 14, 8999.

20 M. D. Horbury, L. A. Baker, W. D. Quan, S. E. Greenough and V. G. Stavros, Phys. Chem. Chem. Phys., 2016, 18, 17691.

21 M. Staniforth and V. G. Stavros, Proc. Math. Phys. Eng. Sci., 2013, 469, 20130458.

22 E. M. M. Tan, M. Hilbers and W. J. Buma, J. Phys. Chem. Lett., 2014, 5, 2464.

23 R. M. Hochstrasser, Acc. Chem. Res., 1968, 1, 266.

24 Y. Miyazaki, Y. Inokuchi, N. Akai and T. Ebata, J. Phys. Chem. Lett., 2015, 6, 1134.

25 X. P. Chang, C. X. Li, B. B. Xie and G. Cui, J. Phys. Chem. A, 2015, 119, 11488.

26 Y. Miyazaki, K. Yamamoto, J. Aoki, T. Ikeda, Y. Inokuchi, M. Ehara and T. Ebata, J. Chem. Phys., 2014, 141, 244313.

27 V. G. Stavros, Nat. Chem., 2014, 6, 955.

28 L. A. Baker, M. D. Horbury, S. E. Greenough, M. N. R. Ashfold and V. G. Stavros, Photochem. Photobiol. Sci., 2015, 14, 1814.

29 L. A. Baker and V. G. Stavros, Sci. Prog., 2016, 99, 282-311.

30 M. D. Horbury, L. A. Baker, W. D. Quan, J. D. Young, M. Staniforth, S. E. Greenough and V. G. Stavros, J. Phys. Chem. A, 2015, 119, 11989.

31 S. E. Greenough, M. D. Horbury, J. O. F. Thompson, G. M. Roberts, T. N. V. Karsili, B. Marchetti, D. Townsend and V. G. Stavros, Phys. Chem. Chem. Phys., 2014, 16, 16187.

32 A. Iqbal, L. J. Pegg and V. G. Stavros, J. Phys. Chem. A, 2008, 112, 9531.

33 U. Even, J. Jortner, D. Noy, N. Lavie and C. Cossart-Magos, J. Chem. Phys., 2000, 112, 8068.

34 S. E. Greenough, G. M. Roberts, N. A. Smith, M. D. Horbury, R. G. McKinlay, J. M. Zurek, M. J. Paterson, P. J. Sadler and V. G. Stavros, Phys. Chem. Chem. Phys., 2014, 16, 19141.

35 L. A. Baker, M. D. Horbury, S. E. Greenough, P. M. Coulter, T. N. V. Karsili, G. M. Roberts, A. J. Orr-Ewing, M. N. R. Ashfold and V. G. Stavros, J. Phys. Chem. Lett., 2015, 6, 1363.

36 M. P. Grubb, A. J. Orr-Ewing and M. N. R. Ashfold, Rev. Sci. Instrum., 2014, 85, 064104.

37 A. S. Chatterley, C. W. West, V. G. Stavros and J. R. R. Verlet, Chem. Sci., 2014, 5, 3963.

38 J. R. Lakowicz, Principles of Fluorescence Spectroscopy, 3rd edn, Springer, Singapore, 2011.

39 L. Blancafirt, V. Ovejas, R. Montero, M. Fernandez-Fernandez and A. Longarte, J. Phys. Chem. Lett., 2016, 7, 1231.

40 T. Ebata, 2016, Private communication.

41 K. Pande, C. D. M. Hutchison, G. Groenhof, A. Aquila, J. S. Robinson, J. Tenboer, S. Basu, S. Boutet, D. P. DePonte, M. Liang, T. A. White, N. A. Zatsepin, O. Yefanov, D. Morozov, 
D. Oberthuer, C. Gati, G. Subramanian, D. James, Y. Zhao, J. Koralek, J. Brayshaw, C. Kupitz, C. Conrad, S. RoyChowdhury, J. D. Coe, M. Metz, P. Lourdu Xavier, T. D. Grant, J. E. Koglin, G. Ketawala, R. Fromme, V. Šrajer, R. Henning, J. C. H. Spence, A. Ourmazd, P. Schwander, U. Weierstall, M. Frank, P. Fromme, A. Barty, H. N. Chapman, K. Moffat, J. J. van Thor and M. Schmidt, Science, 2016, 352, 725.

42 S. Arai and M. C. Sauer Jr, J. Chem. Phys., 1966, 44, 2297.

43 D. S. Larsen, I. H. M. van Stokkum, M. Vengris, M. A. van der Horst, F. L. de Weerd, K. J. Hellingwerf and R. van Grondelle, Biophys. J., 2004, 87, 1858.
44 M. Vengris, I. H. M. van Stokkum, X. He, A. F. Bell, P. J. Tonge, R. van Grondelle and D. S. Larsen, J. Phys. Chem. A, 2004, 108, 4587.

45 K. M. Hanson, S. Narayanan, V. M. Nichols and C. J. Bardeen, Photochem. Photobiol. Sci., 2015, 14, 1607.

46 A. Espagne, D. H. Paik, P. Changenet-Barret, M. M. Martin and A. H. Zewail, ChemPhysChem, 2006, 7, 1717.

47 T. M. Karpkird, S. Wanichweacharungruang and B. Albinsson, Photochem. Photobiol. Sci., 2009, 8, 1455.

48 Y. Nosenko, G. Wiosna-Salyga, M. Kunitski, I. Petkova, A. Singh, W. J. Buma, R. P. Thummel, B. Brutschy and J. Waluk, Angew. Chem., Int. Ed., 2008, 47, 6037. 